\title{
Modeling of Mass Transfer Process in a Fixed Layer of the Adsorbent in Viscous Media
}

\author{
Marat Isakovich Satayev \\ Rustem Feliksovich Altynbekov \\ Abdilla Abibullayevich Saipov \\ Ravshanbek Sultanbekovich Alibekov \\ Mussabekov Akhmetbek Akhylbekovich
}

M. Auezov South Kazakhstan State University, Kazakhstan, Correspondence: Rustem Feliksovich Altynbekov
M. Auezov South Kazakhstan State University, 160012, Shymkent city, Kazakhstan. Email: rustik2030@inbox.ru

Doi:10.5901/ajis.2016.v5n2p151

\begin{abstract}
A mathematical model of adsorption clarification of vegetable oils in a fixed layer of the adsorbent is developed. An equation for the penetration coefficient is proposed based on the extracted component mass transfer regularities analysis depending on the convective flow of a viscous medium, as well as internal diffusion. An equation for calculating the adsorbent layer specific surface area that takes into account connection of the flow through the layer with differential pressure is calculated. A differential equation for the volumetric component flow through the adsorbent layer that takes into account change in the extracted substance concentration is derived on the basis of the theory of mass transfer through porous layers. A solution of this equation allows obtain an expression to determine the coefficient of trapping the layer. An expression to change the trapped component concentration is calculated.
\end{abstract}

Keywords: viscous media, mass transfer process, mathematical model, adsorption clarification, vegetable oils, porous layers.

\section{Introduction}

A vegetable oil, produced by pressing and extraction is a ready-to-eat product. The main problem of food in the world is a lack of protein, which deficit for the universe is about 15 million tons.

An acid-alkali neutralization using sulfuric acid favors to both decoloration of the oil and formation of resinous substances - products of undesirable interaction with triglycerides. Besides, application of expensive sulfuric acid and a large number of alkali for the following oil neutralization increases expenses for its clarification. The preliminary processing of the oil by sulfuric acid allows obtain clear samples of the oil with high decoloration degree (10-15) $\mathrm{mg} / 2$. However, simultaneously with the decoloration in the oil, there is a change in the fat-acid composition in connection with reaction of different components and neutral fat with acid. However, application of activated carbon is available, and, to our opinion, it is a non-toxic method of the oil decoloration. If we consider the main task of the modern production is to use ecologically clean and available raw material, then application of two or several chemicals, duration of the oil settling after carried out clarification stages will allow us express our opinion about advantages of the adsorption clarification of the oils before other methods.

Notwithstanding the fact that the adsorbents in the oil clarification are used from the earliest times and many researches are devoted to this practical and theoretical problem, the generally recognized theory, explaining all phenomena occurring at their contact interaction, has not been developed yet. An adsorption capacity of the adsorbents should be determined taking into account all possible superficial processes and the adsorbents' nature, as well as effect of medium and other factors.

The adsorption in the viscous media is a complex mass transfer process, which behavior depends on properties of the adsorbent and absorbate, on the content of the last in a mixture, temperature, the process behavior hydrodynamic conditions, the layer depth and form, i.e. sizes and structure of apparatus, where the process is carried out. In design and operation of such apparatus, it is necessary to know hydrodynamic filtration regularities of the viscous liquid through the adsorbent fixed layer. It is especially important during operation in the system with the viscous liquids, where application of the fixed layer is possible. 


\section{Literature Review}

Apricot seeds contain $35-40 \%$ of fat, which extraction will allow expand the assortment of food and pharmacopeial oils, as processing of the fruit kernels has not any manufacturing inconveniences for oil and fat enterprises (Jan, 2007; Jan, 2011). Vegetable oils are biologically valuable food products (Vollmann et al., 2010; Lerma García and María Jesús, 2012; Temple et al., 2012; Shewry et al., 1999; David, 2008; Marina Bährle-Rapp, 2007; Tejpal Singh Bisht et al., 2015). However, due to the availability of contaminants in them (coloring and waxy substances, free fatty acids, etc.) (Arutyunyan et al., 2004; Jorge F. et al., 1991), the oils in their natural state cannot meet the requirements for preparation of foodstuff.

Different batch and continuous process flow diagrams for clarification of the oils are used at the oil and fat enterprises (Azimova et al., 2012). They differ in performance and design of the main apparatus for the decoloration and filtration. Our review of clarification processes has found significant shortcomings. The processes of hydration, alkali neutralization, washing and drying do not allow reduce content of the attendant impurities. Therefore, it is necessary to introduce the oil adsorption clarification, which allows carry out deep clarification of the oils from colloid-soluble pigments, wax compounds, free fatty acids and other. The acid-base neutralization forms the basis of the known oil clarification processes (Gupta, 2012; Mounts, 1981; Bhatnagar and Gupta, 1998; Knowles, 1968; Hechi et al., 2009; Kent et al., 2007).

The adsorption refers to the physical and chemical methods of the oil clarification (Edward, 1958; Miyagi et al., 2003). Available data on theoretical and experimental material, devoted to the oil refining and processing study, is poor (Miyagi and Nakajima, 2003; Karabulut et al., 2008; Morgan et al., 1985; Fontanel and Didier, 2011; Mishra et al., 2007; Raju et al., 2014; Ganga et al., 2015). This allows consider the studies on possibilities to produce the refined oil as the task of interest at this time.

The literature review is also indicative of the oil bleaching lability, absence of agreement of opinion on the bleaching mechanism and clarification technology, both in the laboratory and industrial conditions that allows consider this problem as actual.

The literature on the adsorption abounds with design equations, which often do not match with each other. This is due to the difficulties in design of nonstationary process of the adsorption, complexity of its mathematical description, resulted in the fact that the formula used for the engineering design do not always fully correspond to the physical meaning of the described phenomenon. The published studies provide limited information about the features of the adsorption process in the viscous media (Mishra et al., 2007; Raju et al., 2014; Ganga et al., 2015; Raju et al., 2015; Alessandro Delle Site, 2001; Henderson et al., 2015).

Analysis of the experimental and theoretical material on the adsorption in the system of solid phase - viscous liquid showed that the dissolved substance molecules are able to penetrate to the adsorbent grain external surface through the viscous liquid boundary layer practically by means of diffusion. Concentration of the extracted substance at the boundary with the solid body depends on the adsorbed substance molecules diffusion from the adsorbent grain external surface into the channels of its pores. Results obtained in this direction are preliminary (Kapralov et al., 2008; Asnin et al., 2009; Quoc Cuong Do and Seoktae Kang, 2015; Zeid et al., 2013; Ghosh and Reddy, 2013; Wu-Chung Chan and Chia-Wei Cheng, 2012). However, it is the molecules' diffusion rate inside the adsorbent grain, which is determined by its porosity structure, the substance concentration gradient in the direction from the external boundary to the grain center, and the adsorbed substance concentration at the grain's external surface, determines the mass transfer intensity at the adsorption of substances from the viscous liquid phase.

Effect of parameters, characterized by the adsorbent porosity optimal structure, the substance selective adsorption energy from the solution, the molecules diffusion migration in the adsorbent pores, the pore crookedness coefficient, the adsorption space content in the grain pores' total volume should be considered in design and construction of systems for the oil adsorption clarification.

\section{Materials and Methods}

The research objective is to develop the vegetable oils adsorption clarification mathematical model in the adsorbent fixed layer; analyze regularities of the mass transfer and internal diffusion; derive equations for calculating the penetration coefficient, the adsorbent layer specific surface area, the layer trapping coefficient, the trapped component concentration, and differential equation for the volumetric component flow through the adsorbent layer.

In order to study the mass transfer at the oil adsorption clarification using activated carbons, we have used a 
laboratory adsorption plant. In the course of the studies, the following operating conditions such as the flow rate, temperature, time, pressure, concentration were measured.

A complex of classical and modern physical and chemical methods of analysis to determine the oil viscosity, the trapped component concentration has been used to achieve the objective: GOST 30306-95 "Fruit-stone oil and almond oil. Specifications" (State Standard Specification (SSS) GOST 30306-95, 1995).

To determine the diffusion characteristics we have chosen research methods based on the study of different regularities and used analytical equipment such as analyzer of the specific surface area (TriStar 3000, Micromeritics); analyzer of the specific surface area (Sorbi N.4.1, Meta) to determine sorption/desorption isotherms, calculate the porous structure parameters, and measure the porosity by the full adsorption isotherm.

To study the adsorption clarification we have used the modified adsorbent (activated carbon), produced from the apricot kernel shells treated by $\mathrm{ZnCl}_{2}$ with 0,4 impregnation coefficient and the following activation with $\mathrm{CO}_{2}$ at $500^{\circ} \mathrm{C}$ temperature [20].

\section{Results and Discussion}

\subsection{The mass transfer in the adsorbent fixed layer}

The diffusion process of the adsorption can be determined by the following mechanisms: by the substance inlet from the flow core to the solid particles external surface (external diffusion) and the adsorbate transfer inside the particles (internal diffusion). In the limitary situations, one of the mechanisms is limiting. For example, in sufficiently high flow rates or large sizes of the adsorbent grains, the process is limited by the internal diffusion. The adsorption often runs in the intermediate area and determined by both diffusion mechanisms.

The pore structure to a large extent determines the extraction mechanism and rate of its behavior. In through pores, the oil can be moved under the influence of differential pressure, which, as is known, runs at the particle flowaround by the oil stream. At that, the matter solution can be simply displaced from the porous space.

The matter diffusion in the adsorbent pores can be the consequence of different molecule migration character. In sufficiently wide - transport adsorbent pores, the dissolved substance diffusion is carried out in the same way as in the oil free phase. However, calculation of the dissolved substance diffusion rate in the adsorbent grain transport pores is impossible, as a true channel length, by which molecules are diffused under the influence of the concentration gradient to the external grain surface to its center, is uncertain. Taking into account the crookedness and random forms, as well as character of the channels, formed in the adsorbent grain during its preparation, we may state that the real length of the diffusing to the grain center molecules is significantly longer the grain pore effective radius. If we express a ratio of the diffusing molecules true path from the grain external surface to its center to this grain radius in the form of the crookedness coefficient $K_{c r}$., then the dissolved molecules effective diffusion coefficient in the grain $D_{\text {ef. }}(K e l t s e v, 1984)$, calculated for the path along the grain radius, and the true molecular coefficient of the diffusion in the solution, will be related by the next formula:

$$
D_{\text {mol. }}=D_{\text {ef. }} \cdot K_{\text {cr. }}{ }^{2}
$$

The mass transfer in the adsorbent grain is carried out by two ways: the molecules diffusion in the oil, filling the pores, and the adsorbed molecules diffusion in the field of the adsorption forces, action, i.e. in the adsorption space, which in microporous activated carbons is a value close to the volume of micropores. In narrow mesopores, which effective radius only in several times exceeds the diffusing molecules van der Waals sizes, the diffusion is significantly complicated by the fact that in small radius the most of the molecules in their thermal motion face with the pore walls more often than among themselves. Only small part of the molecules, faced with the pore channel wall, is mirrored, as the pore wall surface represents the defects system, comparable to the molecule sizes. The most of the molecules is reflected from the wall in different directions and at different angles (Figure 1). 


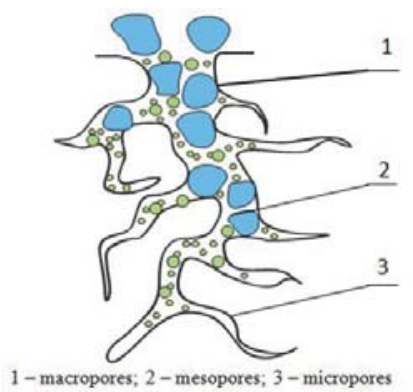

Figure 1 - Scheme of motion of the flows inside the pores.

To perform the transfer efficiency in the system, let's introduce a dissipative function $F$, which represents a sum $J_{i}$ of productions of the component flows by thermodynamic forces (Katchalsky and Curran, 1965; Spigler and Laird, 1980) (Figure 2):

$$
F=\sum J_{i} C_{i}
$$

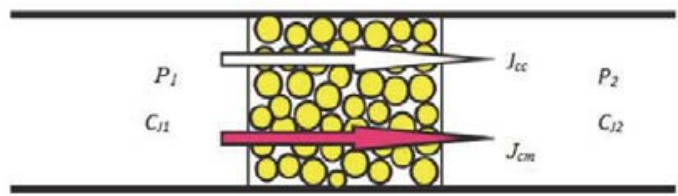

Figure 2 - Scheme of the component flows through the adsorbent layer

In a linear approximation, ${ }_{i}$ flow taking into account cross components can be written in the next form:

$$
J_{i}=\sum_{J} L_{i J} C_{J}
$$

where $L$ - the layer length,

$C$ - the trapped component concentration.

Taking into account that the dissolved substance motion through the layer is caused by availability of difference in chemical potentials $\Delta \mu$ [mi]. Then, neglecting difference in physical potentials, the next formula can be written for the dissipative function (Nakagaki and Takagi, 1986):

$$
F=-J_{S t} \frac{\Delta \mu_{S t}}{L}-J_{C g} \frac{\Delta \mu_{C g}}{L}
$$

where $J_{S t}$ - the supporting component flow,

$J_{C g}-$ the trapped matter flow,

$\Delta \mu_{S t}$ - differences of the chemical potentials for the supporting component,

$\Delta \mu_{\mathrm{cg}}$ - differences of the chemical potentials for the trapped matter.

For the chemical potentials we have:

$\mu=\mu^{0}+R T \ln a$

$\mu_{C g}=\mu_{C g}^{0}+\bar{V} P+R T \ln a_{S}$

where $a$ - chemical activity of the components,

$P$ - pressure,

$T$ - temperature.

The osmotic pressure is related to the supporting component activity by the next correlation:

$$
\Pi=-\frac{R T}{\ln \bar{V}_{S t}} \ln a_{S t}
$$


It follows

$$
\Delta \mu_{S t}=\bar{V}_{S t}(\Delta P-\Delta \Pi)
$$

In turn, it follows from Gibbs-Duhem equation for the two-component system (Nakagaki and Takagi, 1986; Protodyakonov et al, 1982):

$$
\frac{d \mu_{C g}}{d \mu_{S t}}=-\frac{n_{S t}}{n_{C g}}
$$

From the correlations (3) - (9) we obtain:

$$
R T \frac{d\left(\ln a_{C g}\right)}{d \Pi}=\frac{1}{C_{C g}}
$$

where $\mathrm{C}_{\mathrm{cg}}$ - the trapped component concentration.

In a result, we obtain the next expression for the dissipative function in the next form (Nakagaki and Takagi, 1986):

$$
F=-J_{V} \frac{\Delta P}{L}-J_{D} \frac{\Delta \Pi}{L}
$$

where the supporting component volume flow:

$$
J_{V}=\bar{V}_{\mathrm{St}} J_{\mathrm{St}}+\bar{V}_{\mathrm{Cg}} J_{\mathrm{Cg}}
$$

the trapped matter volume flow:

$$
J_{D}=\frac{J_{C g}}{C_{C g}}-\bar{V}_{S t} J_{S t}=u_{C g}-u_{S t}
$$

The correlation (11) can be rewritten in the form of phenomenological equations (Sherwood and Pickford, 1982):

$J_{D}=L_{D P} \Delta P+L_{D} \Delta \pi$;

$J_{V}=L_{P} \Delta P+L_{P D} \Delta \pi$.

This implies a connection between the penetration coefficient and volume flow:

$$
L_{P n}=-L_{P}=-\left.\frac{J_{V}}{\Delta P}\right|_{\Delta \pi=0 .}
$$

The penetration coefficient can be determined from the next correlation:

$$
L_{P n}=\frac{f}{L}\left(\frac{\delta^{2}}{8 \eta}+\frac{\bar{V}_{S t} D_{S t}}{R T}\right) \text {, }
$$

where ${ }^{f}$ - coefficient, connected with the adsorbent layer geometrical characteristic, $\eta$ - viscosity,

$\delta_{-}$the diffusion boundary layer depth.

Let's consider a dripping, occurring by the matter motion by the micropores (Figure 3):

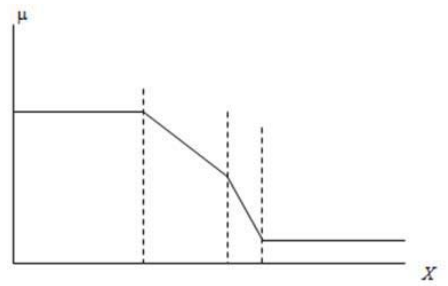

$X$-axis - the layer length $(L, m), Y$-axis - the trapped component chemical potential.

Figure 3 - Change in the trapped component chemical potential along the layer length

\subsection{Hydrodynamic aspects of the vegetable oil adsorption clarification}

Let's connect the filtration rate $V_{F}$, related to the adsorbent surface unit, with $J_{\text {in }}$ flow inside the pores. Let ${ }^{N_{n}}$ - the pores 
number at the surface $S, r_{A v}$ - the pore mean radius. Then it is possible to determine the section coefficient as a relation of the pores full area to the layer full area:

$$
p=\frac{\pi r_{A v}{ }^{2} N_{n}}{S},
$$

Further, in normal conditions, the desired connection is expressed by the next formula (Dytnersky, 1991; Aharony,1985):

$$
V_{F}=p J_{\text {in }},
$$

Q* flow inside the pore is laminar and follows Poiseuille law:

$$
Q^{*}=\frac{\pi r_{A v}{ }^{4}}{8 l \eta} \Delta P \text {. }
$$

where $I$ - the pore length.

The dripping linear rate:

$$
u^{*}=\frac{Q^{*}}{\pi r_{A v}{ }^{2}}
$$

Let's introduce the pore crookedness coefficient concept (Figure 1):

$$
k=\frac{l}{L}
$$

Then, the linear rate expression has the next form:

$$
u^{*}=\frac{r_{A v}^{2}}{8 k L \eta} \Delta P
$$

The porosity coefficient is respectively determined by the next formula:

$$
\varepsilon=p k
$$

If we introduce the adsorbent material true density $\rho_{T}$, we can write an expression for the layer specific surface area:

$$
S_{S p}=\frac{2 \pi r_{A v} l N_{n}}{\rho_{T} L S(1-\varepsilon)}=\frac{2 \varepsilon}{(1-\varepsilon) \rho_{T} r_{A v}}
$$

Hence follows the next correlation:

$$
S_{S p}=\frac{1}{\rho_{T}} \sqrt{\frac{1}{2 k^{2}} \frac{S \Delta P}{L \eta Q} \frac{\varepsilon^{3}}{(1-\varepsilon)^{2}}}
$$

Figure 4 presents the relationship of the layer specific surface area from the porosity calculated by the model. Kozeny constant $K_{K}$ can be introduced into the correlation (21) (Henderson et al., 2015):

$$
K_{\hat{E}}=2 k^{2}
$$

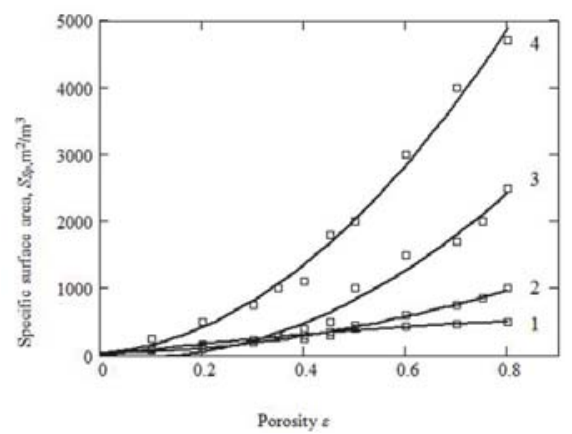

Symbols: points - the experiment; line - calculation by the model. 1 - $\rho_{n} r_{A v}=1000 \mathrm{~kg} / \mathrm{m}^{2} ; 2-580 \mathrm{~kg} / \mathrm{m}^{2} ; 3-220 \mathrm{~kg} / \mathrm{m}^{2} ; 4$ $-130 \mathrm{~kg} / \mathrm{m}^{2}$, where $\rho_{n}$ - the material true density, $\mathrm{kg} / \mathrm{m}^{3} ; r_{A v}$ - the pore mean radius, $\mathrm{m}$.

Figure 4 - Relationship of the layer specific surface area from the porosity 
Now, let's introduce the dripping coefficient by the next formula:

$L_{P n}=\frac{p u^{*}}{\Delta P}=\frac{p k}{L} \frac{r_{A v}{ }^{2}}{8 \eta}$

$p$ value by its character is directly related to the layer porosity:

$p=\frac{\varepsilon}{k}$

From here, we obtain the next expression for the adsorbent layer coefficient:

$f=\frac{\varepsilon}{k^{2}}$

Let's consider the crooked pore, modelling the ordinary channel in the adsorbent layer (Figure 5).

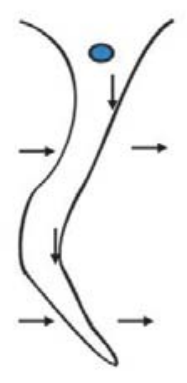

Figure 5 - The adsorbent layer ordinary pore model

On some sections, the motion occurs in the direction perpendicularly to the layer surface. On these sections, ${ }^{f=1}$ value corresponds to the pore section, on the other sections: $f=f_{V}$. Relation of the corresponding lengths of the sections is:

$\frac{\varepsilon}{1-\varepsilon}(46)$.

Further, we have:

$\frac{f_{m}}{f_{V}}=\frac{1}{1-\varepsilon\left(1-f_{V}\right)}$

To consider processes, related to the diffusion transfer in the pores and corresponding phenomenological coefficient, let's modify the transfer equation.

Let's rewrite it in the next form in the beginning:

$J_{D}=\frac{J_{C g}}{C_{C g}}-J_{V}$

For the cross coefficient it can be written as follows:

$L_{D P}=L_{P D}=\sigma L_{P n}$

where $\sigma_{-}$coefficient related to the flow reflection (Nakagaki and Takagi, 1986; Protodyakonov et al., 1982; Sherwood and Pickford, 1982; Dytnersky, 1991; Aharony, 1985; Clement et al., 1985).

Then we obtain:

$\Delta P=\sigma \Delta \Pi-\frac{J_{V}}{L_{P n}}$

It follows:

$J_{C g}=(1-\sigma) \bar{C}_{C g} J_{V}-\omega \Delta \Pi$,

where for $w$ parameter, which characterizes the layer permeability by the trapped component at the expense of the carrier mobility, we have: 


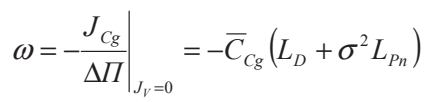

Let's imagine the trapped matter ${ }^{{ }_{C g}}$ in the form of two flows sum:

$J_{C g}=J_{C g}^{(V)}+J_{C g}^{(D)}$

Here

$J_{s}^{(V)}=(1-\sigma) \bar{C}_{s}$

is conditioned by the trapped matter molecules entrainment by the carrier oil molecules, i.e. $(1-\sigma)$ has sense of the layer permeability.

The other component is the diffusion flow in the ordinary sense:

$J_{C g}^{(D)}=-\omega \Delta \Pi$

From her we obtain:

$J_{D}=-\sigma J_{V}+\frac{J_{C g}^{(D)}}{\bar{C}_{C g}^{D}}$

As $J_{C g}$ flow refers to the mass transfer in the absence of the volume flows, it is possible to determine the layer penetration degree as follows (Hentschel and Procaccia, 1984):

$D_{P}=-\frac{J_{C g}^{(D)} L}{\Delta C_{C g}}$

The next correlation is obtained from here:

$D_{P}=\frac{\omega L \Delta \Pi}{\Delta C_{C g}} \approx R T \omega L$

If in some case, by some reasons, it is inconvenient to use the layer depth, then the next nominal coefficient can be introduced:

$D_{P}^{\prime}=\frac{D_{P}}{L}$

Then:

$J_{C g}^{\prime(D)}=-D_{P}^{\prime} \Delta C_{C g}$

Now, substituting:

$\frac{\Delta C}{L}$ to $\frac{d C_{C g}}{d x}$

we obtain the next differential equation, which connects the volume flow with the trapped component concentration change:

$J_{C g}=(1-\sigma) C_{C g} J_{V}-D_{P} \frac{d C_{C g}}{d x}$

Integrating it at the next initial conditions:

$x=0, C_{C g}=C_{H}$

$x=L, C_{C g}=C_{K}$

In a result, we obtain:

$-\frac{(1-\sigma)}{D_{P}} J_{V}=\ln \left[\frac{J_{C g}-(1-\sigma) C_{H} J_{V}}{J_{C g}-(1-\sigma) C_{K} J_{V}}\right]$

As the concentration is connected with the flows by the correlation:

$C_{K}=\frac{J_{C g}}{J_{V}}$

we obtain the calculated expression for the trapped component concentration change: 


$$
\exp \left[-\frac{(1-\sigma) J_{V} L}{D_{P}}\right]=\frac{1}{\sigma}-\frac{1-\sigma}{\sigma} \frac{C_{H}}{C_{K}}
$$

From here, we determine the layer trapping coefficient:

$$
K_{C}=1-\frac{C_{\dot{I}}}{C_{\hat{E}}}
$$

As follows from (48):

$$
K_{C}=\left(\frac{1-\Psi}{1-\sigma \Psi}\right) \sigma
$$

or:

$$
\Psi=\exp \left[-\frac{(1-\sigma) L J_{V}}{D_{P}}\right]
$$

Figure 6 presents representative results of $q$ specific flow calculations.

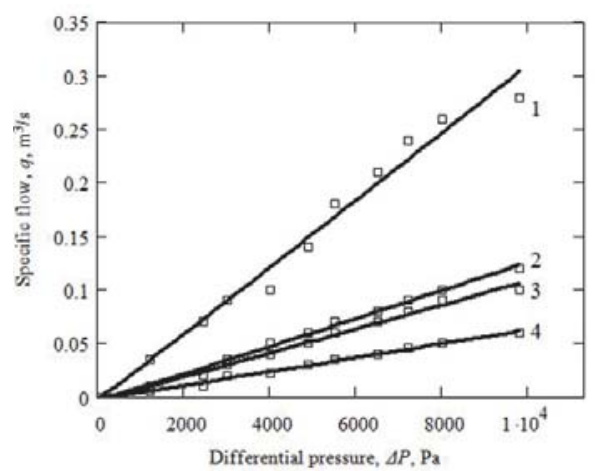

Symbols of the curves: $1-\rho_{n} r_{A v}=1000 \mathrm{~kg} / \mathrm{m}^{2} ; 2-580 \mathrm{~kg} / \mathrm{m}^{2} ; 3-220 \mathrm{~kg} / \mathrm{m}^{2} ; 4-130 \mathrm{~kg} / \mathrm{m}^{2}$, where $\rho_{n}$ - the adsorbent material true density, $\mathrm{kg} / \mathrm{m}^{3} ; r_{A v}$ - the pore mean radius, $\mathrm{m}$, at Kozeny constant $\mathrm{K}=5$ and kinematic viscosity $v=10^{-5} \mathrm{~m}^{2} / \mathrm{s}$.

Figure 6 - The differential pressure - specific flow relationship

The offered mass transfer model, which considers the extracted component mass transfer regularities, viscous medium convective flow, internal diffusion, differential pressure, concentration change at the oil adsorption clarification at the activated carbon fixed layer (equations $13,17,26,28,30,42,45,48$ ), soundly describes the experimental data. It can be recommended as the component in the design procedure for the perspective and effective scheme of the oil clarification in the adsorber with the fixed layer.

\section{Conclusion}

It was established that the diffusion rate of the oil adsorption clarification by the activated carbon is limited by the adsorbate diffusion inside the particles (internal diffusion) and the adsorption in the intermediate field.

On the basis of the mass transfer specified mechanism, the equation to determine the penetration coefficient taking into account the viscous medium convective flow and internal diffusion was offered. The expression, which connects the flow through the layer with the differential pressure and the layer specific flow, that allows calculate the flow, was also offered.

The differential equation, which connects the volume flow with the trapped component concentration change was offered. The expression for the trapping coefficient was obtained in a result of the differential equation solution.

The work was performed by the grant, financed by the Ministry of Education and Science of the Republic of Kazakhstan for 2015-2017 years, contract No. 78-1 from February 12, 2015. Theme: "Development of technology for production of vegetable pharmacopeial oils, using adsorption clarification, which allows reduce energy costs and produce valuable oils with high biological activity". 


\section{References}

Aharony A. (1985). Anomalous diffusion on percolating clusters / Scaling Phenomena in Disordered Systems. Plenum Press.-New. P.289-300.

Arutyunyan N.S. and et al. (2004). Refining oils and fats: Theoretical Foundations, practice, technology and equipment. St. Petersburg: GIORD, $228 p$.

Arysbayeva S.S. Sataeyv, M. I., Shakirov, B. S., Sataeva, L. M., \& Makhalova G.M. . (2007). The method of activated carbon. Pre-patent of Kazakhstan № № 18899

Asnin L.D., Kachmarski K., Fedorov A.A. \& Chekryshkin Y. S. (2009). Description of the dynamics of vapor adsorption in a fixed layer of an adsorbent using various approximations of the mixed-diffusion model. Theoretical Foundations of Chemical Engineering, Vol. 43, No. 3, pp. 260-267.

Azimova S.S., Glushenkova A.I . \& Vinogradova V.I. (2012), Lipids, Lipophilic Components and Essential Oils from Plant Sources. Springer-Verlag London, XII, 992 p.

Bährle-Rapp M. (2007). Apricot Kernel Oil PEG-6 Esters. Springer LexikonKosmetik und Körperpflege, p 43

Bhatnagar A. \& Gupta A. (1998). Chlorpyriphos, Quinalphos, and Lindane Residues in Sesame Seed and Oil (Sesamumindicum L.). Bulletin of Environmental Contamination and Toxicology, Volume 60, Issue 4, pp 596-600

Chen Chen, Hua Zhang, Xuechen Wang. Antibacterial Activated Carbon Fibers Prepared by a New Technology. Modern Applied Science Vol. 2, No. 1

Clement E., Baudet C. \&Hulin J.P. (1985). Multiple scale structure of nonwetting fluid invasion fronts in 3D model porous media. Jour. Phys. Lett. N 46. - L. 1163-1172.

David D. (2008). Brooks bleaching of vegetable oils. Oils and fats, №8, p.11-15.

Dytnersky Y.I. (1991). Basic processes and apparatuses of chemical technology. M .: Chemistry, 493 p.

Edward M. J. (1958). The refining and bleaching of vegetable oils. Journal of the American Oil Chemist's Society, Volume 35, Issue 2, pp 76-83

Fontanel \& Didier. (2011). "Huiles Vegetales Teneur En Matieres Insaponiable", Unsaponifiable Matter in Plant Seed Oils. Originally published in French under the title Lavoisier SAS 978-2-7430-1340-0, 550 p.

Ganga B., Ansari S., Mohamed Y., Ganesh N. V., AK Abdul Hakeem. (2015) MHD radiative boundary layer flow of nanofluid past a vertical platewith internal heat generation / absorption, viscous and ohmic dissipation effects. Journal of the Nigerian Mathematical Society 34 181-194

García L. \& Jesús M. (2012). Characterization and Authentication of Olive and Other Vegetable Oils. New Analytical Methods, Series: Springer Theses, XIX, $218 \mathrm{p}$.

Ghosh R. K. \& Reddy D. D. (2013). Tobacco stem ash as an adsorbent for removal of methylene blue from aqueous solution: equilibrium, kinetics, and mechanism of adsorption. Water Air Soil Pollut 224: 1582

GOST 30306-95 The oil from fruit seeds and almonds. Tehnicheskie usloviya "Fruit-stone oil and almondoil. Specifications"

Gupta, S.K. (2012), Technological Innovations in Major World Oil Crops. Perspectives, Volume 2. X. 322 p.

Hechi E., Amor O. B., Srasra E. \&Zargouni F. (2009). Physico-chemical characterization of acid-activated clay: Its industrial application in the clarification of vegetable oils. Surface Engineering and Applied Electrochemistry, Volume 45, Issue 2, pp 140-144

Henderson N., Brêttas J.C. \& Sacco W. F. (2015). Applicability of the three-parameter Kozeny-Carman generalized equation to the description of viscous fingering in simulations of water flood in heterogeneous porous media. Advances in Engineering Software, Volume 85, Pages 73-80

Hentschel H.G.E. \& Procaccia I. (1984). Relative diffusion in turbulent media: The fractal dimension of clouds. Phys. Rev.A29.-P. 14611470.

J.G. Collin \& Mei P. Lee. Sorption Kinetic Studies of Medical Grade Activated Carbon Prepared from Papaya Seeds. Modern Applied Science Vol. 2, No. 6

Jan W. (2007). Apricot kernel oil. Gooch Encyclopedic Dictionary of Polymers, p 63

Jan W. (2011). Apricot kernel oil. Encyclopedic Dictionary of Polymers, p 46

Jorge F., Toro V.O., Elizabeth G.L., Liliana L. \& Guerrero E. (1991). Adsorption isotherms of squash (Cucurbitamoschata) seed oil on activated carbon. Journal of the American Oil Chemists Society, Volume 68, Issue 8, pp 596-599.

Kapralov P.O., Artemov V.G., Gusev G.A., Tikhonov V.I. \& Volkov A.A. (2008). Diffusion kinetics of water molecules in a nanoporous adsorbent. Bulletin of the Russian Academy of Sciences: Physics, Vol. 72, No. 12, pp. 1679-1683.

Karabulut I., Topcu A., Akmil-Basar C., Onal Y.\& Lampi A. M. (2008). Obtaining Butter Oil Triacylglycerols Free from $\beta$-Carotene and aTocopherol via Activated Carbon Adsorption and Alumina-Column Chromatography Treatments. Journal of the American Oil Chemists' Society, Volume 85, Issue 3, pp 213-219

Katchalsky A. \& Curran P.F. (1965). Non-equilibrium Thermodynamics in Biophysics. Harvard Univ. Press, USA, P. 15-18.

Keltsev N.V. (1984). Fundamentals of adsorption technology. Moscow: Chemistry, 1984. - 453 p.

Kent \& Edmund W. L. (2007). Animal and Vegetable Fats, Oils, and Waxes. Handbook of Industrial Chemistry and Biotechnology, pp 1549-1656

Knowles P. F. (1968). Associations of high levels of Oleic acid in the seed Oil of safflower (carthamustinctorius) with Other plant and seed characteristics. Economic Botany,Volume 22, Issue 2, pp 195-200.

Mishra M., Martin M. \& De Wit A. (2007). Miscible viscous fingering with linear adsorption on the porous matrix. Physics of fluids, 19, 
073101 DOI: 10.1063 / 1.2743610

Miyagi A. \&Nakajima M. (2003). Regeneration of used frying oils using adsorption processing. Journal of the American Oil Chemists' Society, Volume 80, Issue 1, pp 91-96

Miyagi A., Subramanian R. \& Nakajima M. (2003). Membrane and additional adsorption processes for quality improvement of used frying oils. Journal of the American Oil Chemists' Society. Volume 80, Issue 9, pp 927-932

Morgan D. A., Shaw D. B., Sidebottom M. J., Soon T. C. \& Taylor R. S. (1985). The function of bleaching earths in the processing of palm, palm kernel and coconut oils. Journal of the American Oil Chemists' Society., Volume 62, Issue 2, pp 292-299

Mounts T.L., (1981). Chemical and physical effects of processing fats and oils. Journal of the American Oil Chemists' Society., Volume 58, Issue 1, pp 51A-54A

Nakagaki M. \& Takagi R. (1986). Membrane and Membrane Processes. Plenum Press.New York, London. P.77-84.

Protodyakonov I.O., Martsulevich N.A.\& Markov A.V. (1982). Transport phenomena in the processes of chemical tehnologii. L: Chemistry. 695p.

Quoc Cuong Do \& Seoktae Kang (2015). Thermodynamic analysis of fatty acid harvesting by novel carbon-based adsorbent. Environ SciPollut Res, DOI 10.1007 / s11356-015-4428-4

Raju K.V.S., Sudhakar R.T., Raju M.C., Satya Narayana P.V. \& Venkataramana S. (2014). MHD convective flow through porous mediumin a horizontal channel with insulated and impermeable bottom wall in the presence of viscous dissipation and Joule heating. Ain Shams Engineering Journal 5, 543-551.

Raju M.C., Varma S.V.K. \& Seshaiah B. (2015). Heat transfer effects on a viscous dissipative fluid flow past a vertical plate in the presence of induced magnetic field. Ain Shams Engineering Journal 6, 333-339.

S. Syafalni, Ismail Abustan, Aderiza Brahmana, Siti Nor Farhana Zakaria1 \& Rohana Abdullah. Peat Water Treatment Using Combination of Cationic Surfactant Modified Zeolite, Granular Activated Carbon, and Limestone. Modern Applied Science; Vol. 7 , No. 2; 2013, doi:10.5539/mas.v7n2p39

S.Senthilkumaar, S.K. Krishna, P. Kalaamani, C. V. Subburamaan, N. Ganapathi Subramaniam. Adsorption of Organophosphorous Pesticide from Aqueous Solution Using "Waste" Jute Fiber Carbon. Modern Applied Science Vol. 4, No. 6

Satayev, M., Shakirov, B., Mutaliyeva, B., Satayeva, L., Altynbekov, R., Baiysbay, O., \& Alibekov R. (2012). Mathematical modeling of methoxyanabasine C11H16N2O polymer solution ultrafiltration. Heat Mass Transfer, 48(6), 979-987. http://dx.doi.org/10.1007/ s00231-011-0948-8

Sherwood T. \& Pickford R. (1982). Massoperedacha. M .: Himiya. 695p.

Shewry, Peter R ., Casey, R. (1999). Seed Proteins, 1016 p.

Site A. D. (2001). Factors affecting sorption of organic compounds in natural sorbent / water systems and sorption coeffients for selected pollutants. A Review »J. Phys. Chem. Ref. Data, Vol. 30, No. 1,

Spigler K.S. \& Laird A.D.K. (1980). Principles of desalination. Academic Press.-New York. P. 26-29.

Syafalni S., Ismail Abustan, Irvan Dahlan, \& Chan Kok Wah, Genius Umar. Treatment of Dye Wastewater Using Granular Activated Carbon and Zeolite Filter. Modern Applied Science Vol. 6, No. 2; doi:10.5539/mas.v6n2p37

Tejpal S. B., Satish K. S., Ramesh C. S., Virendra K. R., Vijay K. Y., Anil K. D., Ashok K. S. \& Chandra S. C. (2015). Improvement of efficiency of oil extraction from wild apricot kernels by using enzymes. Journal of Food Science and Technology DOI 10.1007 I s13197-013-1155-z

Temple, Norman J .; Wilson, Ted; Jacobs, David R. (2012). Nutritional Health, Strategies for Disease Prevention. Series: Nutrition and Health. 3rd ed., XXI, 559 p.

Vollmann, Johann, Rajcan \& Istvan. (2010). Oil Crops. Series: Handbook of Plant Breeding. Vol. 4, , XVI, 548 p.

Wu-Chung Chan \& Chia-Wei Cheng (2012). Mass transport process for adsorption of metal ions onto a new type adsorbent poly (vinyl alcohol) (PVA) / peat / clay composite bead. JPolymRes 19: 9964

Zeid A. A. \& Mu N., Rahmat A. (2013). Kinetic, equilibrium isotherm and thermodynamic studies of Cr (VI) adsorption onto low-cost adsorbent developed from peanut shell activated with phosphoric acid. Environ SciPollut Res 20: 3351-3365 
